A. Osada

Nagoya Math. J.

Vol. 57 (1974), 163-170

\title{
ON A PROBLEM OF BONAR CONCERNING FATOU POINTS FOR ANNULAR FUNCTIONS
}

\author{
AKIO OSADA
}

The purpose of this paper is to study the distribution of Fatou points of annular functions introduced by Bagemihl and Erdös [1]. Recall that a function $f(z)$, regular in the open unit disk $D:|z|<1$, is referred to as an annular function if there exists a sequence $\left\{J_{n}\right\}$ of closed Jordan curves, converging out to the unit circle $C:|z|=1$, such that the minimum modulus of $f(z)$ on $J_{n}$ increases to infinity. If the $J_{n}$ can be taken as circles concentric with $C, f(z)$ will be called strongly annular.

As a direct consequence of the definition, an annular function $f(z)$ can have at most one Fatou point, i.e., a point on $C$ at which $f(z)$ has an angular limit, on any subarc of $C$ which has no limit points of zeros of $f(z)$ and the Fatou value of $f(z)$, i.e., the angular limit, must be the point at infinity $\infty$. A natural question arises concerning to this observation: Does there really exist a Fatou point for every annular function? Bagemihl and Erdös [1] constructed an example of a strongly annular function which has no Fatou points. Many examples of annular functions are considered in Bonar [3], but it is not clear whether they have Fatou points or not. For these reasons Bonar [3] asks whether the value $\infty$ is a Fatou value for some annular function. The aim of this paper is to give an affirmative answer to this question of Bonar.

We shall recall first some elementary properties of annular functions. Every annular function $f(z)$ has infinitely many zeros and consequently their limit points form a non-empty closed subset, say $Z^{\prime}(f)$, of $C$. We know that the set $Z^{\prime}(f)$ coincides with the full circle $C$ for almost every $f(z)$ of well known examples of annular functions. But as is shown in [2] and [5] there really exists an annular function $f(z)$ such that $Z^{\prime}(f)$ does not coincide with $C$. For such $f(z)$, the complement of $Z^{\prime}(f)$ on $C$ consists of at most a countable number of disjoint open arcs. How-

Received June 7, 1974. 
ever, the annularity of $f(z)$ forbids any of them to contain more than one Fatou point of $f(z)$. Observation of this simple fact suggests us that a modification of the technique used in our former paper [5] yields the following result:

THEOREM. For an arbitrary pair of two distinct points $\zeta_{1}$ and $\zeta_{2}$ on $C$, there exists a strongly annular function $f(z)$ with $\zeta_{1}$ as the only limit point of its zeros and with $\zeta_{2}$ as its only Fatou point.

Proof. The idea of our proof is originally due to Barth and Schneider [2]. Additionally, making an essential use of the reflection principle for harmonic functions, we shall obtain the required function $f(z)$ by imposing some additional conditions on the example of the strongly annular function constructed in [5].

For simplicity we shall consider the case where $\zeta_{1}=-1$ and $\zeta_{2}=1$. First choose a sequence $\left\{a_{n}\right\}$ of real numbers with the following two properties :

$$
\begin{gathered}
\frac{\sqrt{2}}{2}<a_{n} \uparrow 1, a_{n}^{2}>a_{n-1} a_{n+1} ; \\
\sum\left(\cos ^{-1} a_{n}\right)^{2}=+\infty, \quad \sum\left(\cos ^{-1} a_{n}\right)^{3}<+\infty, \quad 0<\cos ^{-1} a_{n}<\frac{\pi}{4} .
\end{gathered}
$$

For example, $\{(n+2) /(n+3)\}$ is such one. Next we shall make some technical definitions. Given $0 \leq a<b \leq+\infty$ and $0<t<\pi$, denote by $D(a, b ; t)$ the annular sector

$$
\{z ; a<|z|<b \text { and }-t<\arg z<t\},
$$

Moreover, for $0<a<+\infty$ and $-\pi \leq t_{1}<t_{2} \leq \pi$, we consider circular ares

$$
\sigma\left(a ; t_{1}, t_{2}\right)=\left\{z ;|z|=a \text { and } t_{1} \leq \arg z \leq t_{2}\right\}
$$

and

$$
\sigma_{0}\left(a ; t_{1}, t_{2}\right)=\left\{z ;|z|=a \text { and } t_{1}<\arg z<t_{2}\right\} .
$$

If we set $\cos ^{-1} a_{n}=t_{n}$, then since $a_{n} \uparrow 1, \pi / 4>t_{n} \downarrow 0$. Relative to this sequence $\left\{t_{n}\right\}$, we take three more sequences $\left\{\varepsilon_{n}\right\},\left\{t_{n}^{\prime}\right\}$ and $\left\{t_{n}^{\prime \prime}\right\}$ of real numbers such that

$$
0<\varepsilon_{n}<t_{n}^{2}, \quad \sum \varepsilon_{n}<+\infty ;
$$




$$
t_{n}<t_{n}^{\prime}<t_{n}^{\prime \prime}<\min \left(\pi t_{n}, t_{n-1}\right), t_{0}=\frac{\pi}{4}
$$

As the first step of our argument, we consider a real-valued function $p_{1}(t)$ on the closed interval $[-\pi, \pi]$ such that

(5) $\quad p_{1}(t)=2 t_{1}^{2} \quad\left(t \in\left[-t_{1}^{\prime}, t_{1}^{\prime}\right]\right), \quad p_{1}(t)=\varepsilon_{1} \quad\left(t \in\left[-\pi,-t_{1}^{\prime \prime}\right] \cup\left[t_{1}^{\prime \prime}, \pi\right]\right)$;

$$
p_{1} \in C^{1}(-\pi, \pi), \quad \varepsilon_{1} \leq p_{1}(t) \leq 2 t_{1}^{2} \quad\left(t \in\left[-t_{1}^{\prime \prime},-t_{1}^{\prime}\right] \cup\left[t_{1}^{\prime}, t_{1}^{\prime \prime}\right]\right) .
$$

Using this function as boundary values, we consider the function

$$
F_{1}(z)=\frac{1}{2 \pi} \int_{-\pi}^{\pi} p_{1}(t) \frac{\zeta+z}{\zeta-z} d t \quad\left(\zeta=a_{1} e^{i t},|z|<a_{1}\right)
$$

which is obviously regular in $|z|<a_{1}$ and must be continuous on the closed disk $|z| \leq a_{1}$ because of condition (5) and the continuous differentiability of $p_{1}(t)$ (see [4], p. 78-79). Moreover, since $p_{1}(t)=2 t_{1}^{2}$ for every $t$ on $\left[-t_{1}^{\prime}, t_{1}^{\prime}\right], F_{1}(z)$ may be continued analytically into the annular sector $D\left(a_{1}, \infty ; t_{1}^{\prime}\right)$ across the open arc $\sigma_{0}\left(a_{1} ;-t_{1}^{\prime}, t_{1}^{\prime}\right)$ by virtue of the reflection principle. We use the same notation $F_{1}(z)$ to denote this extended function. Consequently it is regular in the region

$$
R_{1}=\left\{|z|<a_{1}\right\} \cup \sigma_{0}\left(a_{1} ;-t_{1}^{\prime}, t_{1}^{\prime}\right) \cup D\left(a_{1}, \infty ; t_{1}^{\prime}\right)
$$

and hence particularly is also regular in the region

$$
D_{1}=\left\{|z|<a_{1}\right\} \cup \sigma_{0}\left(a_{1} ;-t_{1}, t_{1}\right) \cup D\left(a_{1}, 1 ; t_{1}\right)
$$

and is continuous on $\bar{D}_{1}$, the closure of $D_{1}$ in the $z$-plane. In order to apply the Mergelyan approximation theorem, we need one more sequence $\left\{\theta_{n}\right\}$ of real numbers such that

$$
\frac{\pi}{2}<\theta_{n} \uparrow \pi, \quad \tan \frac{\pi-\theta_{n}}{2}<\frac{a_{n+2}-a_{n+1}}{a_{n+2}+a_{n+1}}, \quad \frac{\pi}{6}+\sin ^{-1} \frac{1}{2 a_{n+2}} \leq \theta_{n}
$$

where $\pi / 2<\sin ^{-1} a<\pi$ for $0<a<1$. The last inequality of (7) guarantees that the annular sector

$$
\left\{z \in D ; a_{n-1}<|z|<a_{n+1}, \theta_{n_{-1}}<\arg z \leq \pi \text { or }-\pi \leq \arg z<-\theta_{n-1}\right\}
$$

is contained in the interior $T$ of the triangle whose vertices are $i / \sqrt{3}$, $-i / \sqrt{\mathbf{3}}$ and -1 . Here we take two closed arcs $\sigma\left(a_{2} ; t_{1}, \theta_{2}\right)$ and $\sigma\left(a_{2} ;-\theta_{2},-t_{1}\right)\left(\sigma_{2}, \sigma_{2}^{\prime}\right.$ in notation), and join them with $\bar{D}_{1}$ to obtain a compact set $K_{1}=\bar{D}_{1} \cup \sigma_{2} \cup \sigma_{2}^{\prime}$, on which we shall define a continuous 
function $G_{1}(z)$ in such a way that

$$
\begin{gathered}
G_{1}(z)=F_{1}(z) \quad\left(z \in \bar{D}_{1}\right) ; \\
G_{1}(z)=F_{1}\left(a_{2} e^{i t_{1}}\right) \quad\left(z \in \sigma_{2}\right), \quad G_{1}(z)=F_{1}\left(a_{2} e^{-i t_{1}}\right) \quad\left(z \in \sigma_{2}^{\prime}\right) .
\end{gathered}
$$

Evidently we can apply the Mergelyan theorem to this pair of $K_{1}$ and $G_{1}(z)$ and consequently can find a polynomial $g_{1}(z)$ such that

$$
\left|G_{1}(z)-g_{1}(z)\right|<\varepsilon_{1} \quad\left(z \in K_{1}\right) .
$$

Therefore, together with (4), (5) and (6), this inequality implies that

$$
\operatorname{Re} g_{1}(z)>0\left(|z|<a_{1}\right), \quad \operatorname{Re} g_{1}(0)<2\left(t_{1}^{3}+\varepsilon_{1}\right) .
$$

Moreover, since $\varepsilon_{1}<t_{1}^{2}$ by (3) and $\operatorname{Re} F_{1}(z)>2 t_{1}^{2}$ for $z \in D\left(a_{1}, \infty ; t_{1}^{\prime}\right)$ by the definition of $p_{1}(t)$, it is easy to see that

$$
\operatorname{Re} g_{1}(z)>t_{1}^{2} \quad\left(z \in D\left(a_{1}, 1 ; t_{1}\right) \cup \sigma_{2} \cup \sigma_{2}^{\prime}\right) .
$$

Now we shall construct polynomials $\left\{g_{n}(z)\right\}$ inductively as follows:

$$
\begin{gathered}
\operatorname{Re} g_{n}(z)>0\left(|z|<a_{n}\right), \quad \operatorname{Re} g_{n}(0)<2\left(t_{n}^{3}+\varepsilon_{n}\right) ; \\
\operatorname{Re} g_{n}(z)>t_{n}^{2}\left(z \in D\left(a_{n}, 1 ; t_{n}\right)\right) ; \\
\sum_{j=1}^{n} \operatorname{Re} g_{j}(z)>\sum_{j=1}^{n} t_{j}^{2}\left(z \in \sigma_{n+1} \cup \sigma_{n+1}^{\prime}\right),
\end{gathered}
$$

where $\sigma_{n+1}=\sigma\left(a_{n+1} ; t_{n}, \theta_{n+1}\right)$ and $\sigma_{n+1}^{\prime}=\sigma\left(a_{n+1} ;-\theta_{n+1},-t_{n}\right)$. As in the first step, we again consider a real-valued function $p_{n}(t)$ on the closed interval $[-\pi, \pi]$ such that

$\left(5^{\prime}\right) \quad p_{n}(t)=2 t_{n}^{2} \quad\left(t \in\left[-t_{n}^{\prime}, t_{n}^{\prime}\right]\right), \quad p_{n}(t)=\varepsilon_{n} \quad\left(t \in\left[-\pi,-t_{n}^{\prime \prime}\right] \cup\left[t_{n}^{\prime \prime}, \pi\right]\right) ;$

$$
p_{n} \in C^{1}(-\pi, \pi), \quad \varepsilon_{n} \leq p_{n}(t) \leq 2 t_{n}^{2} \quad\left(t \in\left[-t_{n}^{\prime \prime},-t_{n}^{\prime}\right] \cup\left[t_{n}^{\prime}, t_{n}^{\prime \prime}\right]\right) .
$$

Using $p_{n}(t)$ as boundary values, we also define the function $F_{n}(z)$ by the relation

$$
F_{n}(z)=\frac{1}{2 \pi} \int_{-\pi}^{\pi} p_{n}(t) \frac{\zeta+z}{\zeta-z} d t \quad\left(\zeta=a_{n} e^{i t},|z|<a_{n}\right) .
$$

For almost the same reasons as before, $F_{n}(z)$ is obviously regular in the disk $|z|<a_{n}$ and must be continuous on the closed disk $|z| \leq a_{n}$; and furthermore can be continued analytically into $D\left(a_{n}, \infty ; t_{n}^{\prime}\right)$ across $\sigma_{0}\left(a_{n} ;-t_{n}^{\prime}, t_{n}^{\prime}\right)$. This time, too, we shall use the same notation $F_{n}(z)$ for 
this extended function which is, by virtue of the reflection principle, regular in the region

$$
R_{n}=\left\{|z|<a_{n}\right\} \cup \sigma_{0}\left(a_{n} ;-t_{n}^{\prime}, t_{n}^{\prime}\right) \cup D\left(a_{n}, \infty ; t_{n}^{\prime}\right) .
$$

In particular, $F_{n}(z)$ is also regular in the region

$$
D_{n}=\left\{|z|<a_{n}\right\} \cup \sigma_{0}\left(a_{n} ;-t_{n}, t_{n}\right) \cup D\left(a_{n}, 1 ; t_{n}\right)
$$

and must be continuous on its closure $\bar{D}_{n}$. In order to make a crucial use of Mergelyan's theorem again, we shall prepare a compact set $K_{n}$ whose complement is connected and a function $G_{n}(z)$ which is continuous on $K_{n}$ and is regular in its interior. As $K_{n}$, we use the compact set $\bar{D}_{n} \cup \sigma_{n+1} \cup \sigma_{n+1}^{\prime}$. Next, keeping in mind that

$$
\sigma_{n+1}=\sigma\left(a_{n+1} ; t_{n}, t_{n-1}\right) \cup \sigma\left(a_{n+1} ; t_{n-1}, \theta_{n+1}\right)
$$

and

$$
\sigma_{n+1}^{\prime}=\sigma\left(a_{n+1} ;-\theta_{n+1},-t_{n-1}\right) \cup \sigma\left(a_{n+1} ;-t_{n-1},-t_{n}\right),
$$

we shall define $G_{n}(z)$ as follows:

$$
\begin{gathered}
G_{n}(z)=F_{n}(z) \quad\left(z \in \bar{D}_{n}\right) ; \\
G_{n}(z)=w_{n}+\frac{t-t_{n}}{t_{n-1}-t_{n}}\left(2 \alpha_{n}-w_{n}\right), \quad w_{n}=F_{n}\left(a_{n+1} e^{i t_{n}}\right) \\
\left(z=a_{n+1} e^{i t}\left(t_{n} \leq t \leq t_{n-1}\right)\right) \\
G_{n}(z)=2 \alpha_{n}\left(z \in \sigma\left(a_{n+1} ; t_{n-1}, \theta_{n+1}\right)\right) ; \\
G_{n}(z)=w_{n}^{\prime}+\frac{t+t_{n}}{t_{n}-t_{n-1}}\left(2 \alpha_{n}-w_{n}^{\prime}\right), \quad w_{n}^{\prime}=F_{n}\left(a_{n+1} e^{-i t_{n}}\right) \\
\left(z=a_{n+1} e^{i t}\left(-t_{n-1} \leq t \leq-t_{n}\right)\right) \\
G_{n}(z)=2 \alpha_{n}\left(z \in \sigma\left(a_{n+1} ;-\theta_{n+1},-t_{n-1}\right)\right)
\end{gathered}
$$

where

$$
\begin{aligned}
\alpha_{n}= & \sum_{j=1}^{n} t_{j}^{2}+\sum_{j=1}^{n-1} \max \left\{\left|\operatorname{Re} g_{j}(z)\right| ; z \in \sigma\left(a_{n+1} ; t_{n_{-1}}, \theta_{n_{+1}}\right)\right. \\
& \left.\cup \sigma\left(a_{n_{+1}} ;-\theta_{n_{+1}},-t_{n_{-1}}\right)\right\} .
\end{aligned}
$$

The role of $\alpha_{n}$ is to make $\operatorname{Re} G_{n}(z)$ fairly large on $\sigma_{n+1} \cup \sigma_{n+1}^{\prime}$. Applying the Mergelyan theorem to the pair of $K_{n}$ and $G_{n}(z)$, we can find a polynomial $g_{n}(z)$ such that 


$$
\left|G_{n}(z)-g_{n}(z)\right|<\varepsilon_{n} \quad\left(z \in K_{n}\right) .
$$

Since $G_{n}(z)=F_{n}(z)\left(z \in \bar{D}_{n}\right)$ by $\left(8^{\prime}\right)$ and $\varepsilon_{n}<t_{n}^{2}$ by (3), using (16) and recalling the definition of $p_{n}(t)$ stated in $\left(5^{\prime}\right)$ and $\left(6^{\prime}\right)$, we can easily show that $g_{n}(z)$ has property (12). Furthermore, it follows from the reflection principle for harmonic functions that

$$
\operatorname{Re} F_{n}(z)>2 t_{n}^{2} \text { for every } z \in D\left(a_{n}, \infty ; t_{n}^{\prime}\right) \text {. }
$$

Therefore, using (3), (8') and (16), we can conclude that (13) is valid. As for property (14), first note that the arc $\sigma_{n+1}$ is divided into two closed arcs $\sigma\left(a_{n+1} ; t_{n}, t_{n-1}\right)$ and $\sigma\left(a_{n+1} ; t_{n-1}, \theta_{n+1}\right)$, and that the former is included in $D\left(a_{j}, 1 ; t_{j}\right)$ for each $j(1 \leq j \leq n-1)$. For such $j$, it follows from (13) that

$$
\operatorname{Re} g_{j}(z)>t_{j}^{2}
$$

for $z \in D\left(a_{j}, 1 ; t_{j}\right)$ and particularly for $z \in \sigma\left(a_{n+1} ; t_{n}, t_{n-1}\right)$. On the other hand, by virtue of $\left(9^{\prime}\right)$ and (15), we have that

$$
\text { Re } g_{n}(z)>t_{n}^{2} \quad \text { for } \quad z \in \sigma\left(a_{n+1} ; t_{n}, t_{n-1}\right) \text {. }
$$

Moreover, since $G_{n}(z)=2 \alpha_{n}$ for every $z \in \sigma\left(a_{n+1} ; t_{n-1}, \theta_{n-1}\right)$, making use of (15) and (16), we can conclude that (14) surely holds for every $z \in \sigma_{n+1}$. Similarly it is shown that the same also holds for each $z \in \sigma_{n+1}^{\prime}$. We finally remark that, since $\operatorname{Re} g_{j}(z)>t_{j}^{2}$ in $D\left(a_{j}, 1 ; t_{j}\right)$ for $1 \leq j \leq n$, (14) also holds for $z \in D\left(a_{n}, 1 ; t_{n}\right)$.

Once the polynomials $\left\{g_{n}(z)\right\}$ have been constructed as above, the remainder of the proof is fairly easy. To begin with, consider the series $P(z)=\sum \operatorname{Re} g_{n}(z)$. Then, by virtue of the Harnack principle, it follows from (2), (3) and (12) that $P(z)$ uniformly converges on any compact subset of $D$ and hence defines a harmonic function there. As usual, we consider a harmonic function $Q(z)$ conjugate to $P(z)$ in the open unit disk $D$ and set

$$
\exp \{P(z)+i Q(z)\}=g(z) .
$$

Needless to say, $g(z)$ is regular and non-zero in $D$. If we denote by $A_{n}$ the closed arc $\sigma\left(a_{n} ;-\theta_{n}, \theta_{n}\right)$ for each $n$, by virtue of the fact that

$$
A_{n+1} \subset D\left(a_{n}, 1 ; t_{n}\right) \cup \sigma_{n+1} \cup \sigma_{n+1}^{\prime}
$$

and 


$$
D\left(a_{n}, a_{n+1} ; t_{n}\right) \subset D\left(a_{n}, 1 ; t_{n}\right),
$$

using (12), (13) and (14), we can show that

$$
|g(z)| \geq \exp \sum_{j=1}^{n} t_{j}^{2} \quad\left(z \in A_{n+1} \cup D\left(a_{n}, a_{n+1} ; t_{n}\right)\right) .
$$

On the other hand, taking (7) into account and using the procedure employed in [5], we can construct a function $h(z)$, regular in $D$, such that

$$
\begin{gathered}
|h(z)|>\frac{1}{2} \quad(z \in D \cap\{\operatorname{Re} z>0\}) ; \\
\{z \in D ; h(z)=0\} \subset T ; \\
|g(z) h(z)| \geq \frac{1}{2} \exp \sum_{j=1}^{n} t_{j}^{2} \quad\left(|z|=a_{n+1}\right) .
\end{gathered}
$$

Consider the function

$$
f(z)=g(z) h(z) .
$$

Then, it follows from (17),(18) and (20) that, for each $n$,

$$
\begin{aligned}
& |f(z)| \geq \frac{1}{2} \exp \sum_{j=1}^{n} t_{j}^{2} \\
& \quad \text { for every } z \text { of }\left\{z \in D ;|z|=a_{n_{+1}}\right\} \cup D\left(a_{n}, a_{n+1} ; t_{n}\right) .
\end{aligned}
$$

Therefore, in view of the fact that

$$
\left\{z \in D ;\left|z-\frac{1}{2}\right|<\frac{1}{2} \text { and } \operatorname{Re} z>a_{1}\right\} \subset \bigcup_{n=1}^{\infty} \overline{D\left(a_{n}, a_{n+1} ; t_{n}\right)}
$$

and $\sum_{j=1}^{n} t_{j}^{2} \rightarrow+\infty$, (19) and (21) imply that the function $f(z)$ has the properties stated in Theorem in the case where $\zeta_{1}=-1$ and $\zeta_{2}=1$. As can be seen by examing the above proof, we can conclude that our theorem also holds for any pair of distinct $\zeta_{1}$ and $\zeta_{2}$ on $C$.

\section{REFERENCES}

[1] F. Bagemihl and P. Erdös, A problem concerning the zeros of a certain kind of holomorphic function in the unit disk, J. Reine Angew. Math. 214/215 (1964), $340-344$.

[2] K. Barth and W. Schneider, On a problem of Bagemihl and Erdös concerning the distribution of zeros of an annular function, J. Reine Angew. Math. 234 (1969), 179-183. 
[ 3 ] D. D. Bonar, On annular functions, Springer, Berlin, 1971.

[ 4 ] K. Hoffman, Banach spaces of analytic functions, Prentice-Hall, Inc., 1962.

[5] A. Osada, On the distribution of zeros of a strongly annular function, Nagoya Math. J. (to appear).

Gifu College of Pharmacy 